\title{
Meeting Acute Migraine Treatment Needs through Novel Treatment Formulations
}

\author{
Stephen D. Silberstein \\ Jefferson Headache Center, Thomas Jefferson University Hospital, Philadelphia, Pennsylvania 19107
}

\begin{abstract}
Summary: Migraineurs often do not use acute migraine-specific medications. Patient-reported satisfaction with triptans is modest. Patients are generally interested in obtaining more rapid and complete symptom relief. The role of trigeminal vascular activation may explain why some patients fail to respond to current treatment. Novel formulations of currently available acute migraine treatments have been developed, with improved clinical outcomes, response times, and pain-free rates. Currently available effective,
\end{abstract}

novel, acute migraine therapies include needle-free injectable sumatriptan and effervescent diclofenac. Orally inhaled dihydroergotamine is a new treatment modality. These novel formulations may help patients achieve desirable outcomes, including faster and more complete relief, more consistent response, and improved drug tolerability. Key Words: Dihydroergotamine, effervescent, iontophoresis, inhalation treatment, needle-free, pain-free, triptan.

\section{INTRODUCTION}

Despite the development of new migraine-specific therapies, migraine patients still have unmet needs. The American Migraine Prevalence and Prevention Study reported that migraine-specific acute treatment was routinely used by only $19 \%$ of migraineurs. The most used treatment was triptans, with ergots used by $<1 \%$ of patients. ${ }^{1}$ Migraine sufferers continue to rely on overthe-counter preparations $(68 \%)$. Opioids are used by $11 \%$ of patients and butalbital by $6 \%$. Why do only a minority of patients use migraine-specific prescription medications? This can be explained by patient dissatisfaction with currently available specific treatments.

Satisfaction with current acute therapies was evaluated by the Patient Perception of Migraine Questionnaire. ${ }^{2}$ Satisfaction was rated for acute medication attributes, including efficacy, speed of relief, and consistency of benefit, using a 7-point Likert scale, ranging from 1 (very dissatisfied) to 7 (very satisfied). Each satisfaction item scored an average of 5 (representing somewhat satisfied), with only ease of use and convenience items scoring moderately satisfied. No item achieved a rating of very satisfied. When migraineurs are questioned regarding

Address correspondence and reprint requests to: Stephen D. Silberstein, M.D., Jefferson Headache Center, Thomas Jefferson University Hospital, 111 S. 11th Street, Suite 8130, Philadelphia, PA 19107. E-mail: stephen.silberstein@ jefferson.edu. factors that influence acute therapy satisfaction, achieving pain relief in $\leq 1 \mathrm{~h}$ and obtaining complete symptom relief within $2 \mathrm{~h}$ were linked to enhanced satisfaction. ${ }^{3}$

Why do many current acute therapies fail? New information on migraine pathophysiology can be used to enhance therapy. Novel formulations of acute migraine treatments have been developed to improve patient-preferred outcomes. This article will review new information on drug limitations and migraine pathogenesis, and present data for new acute therapies that are currently available and those that will likely become available in the near future.

\section{LIMITATIONS OF TRADITIONAL ACUTE MIGRAINE TREATMENTS}

Patients often do not respond to triptan therapy. A review of efficacy data for traditional triptan formulations showed that as many as $40 \%$ of attacks and $25 \%$ of migraine patients were triptan nonresponders. ${ }^{4} \mathrm{~A}$ more recent study testing the response of 105 migraine patients to six different triptans reported a clinical response by $71 \%$ of patients for at least two of three consecutive migraine attacks. ${ }^{5}$ These data support that alternative therapies will be needed for as many as one in three migraineurs.

Drug-delivery formulations can limit drug effectiveness and patient satisfaction. Although patients often 
prefer the convenience of oral therapy, ${ }^{6}$ associated nausea, vomiting, and gastric stasis limit their effectiveness. Migraineurs have delayed gastric emptying during and between migraine attacks. ${ }^{7}$ In one study, three migraineurs ingested a standard meal, followed by gastric scintigraphy performed during three conditions: 1) a spontaneous migraine, 2) a visually-induced migraine, and 3) an interictal period in which no migraine occurred for 3 days before and 3 days after testing. Time to halfemptying was delayed during both migraine attacks and interictally (112 mins for controls, 124 mins with spontaneous migraine, 182 mins with induced migraine, and 243 mins between attacks). Similarly, the amount of radioactive material remaining $2 \mathrm{~h}$ after ingestion was greater in migraineurs (44\% in controls vs. $51-64 \%$ in migraine conditions). These data provide additional evidence for delayed gastric motility in migraineurs.

\section{MIGRAINE PATHOPHYSIOLOGY SUGGESTS IMPORTANT TREATMENT TARGETS}

Migraine may be initiated by neuroinflammation of dural and meningeal trigeminal nociceptors, sensitizing peripheral neurons ${ }^{8}$ and subsequently resulting in central trigeminal nucleus caudalis hyperexcitability. ${ }^{8}$ Although migraine is often considered a neuronal disorder, glia exert important modulatory influences that affect migraine pathophysiology. Glia influence neuronal excitability by setting neuronal activation thresholds. Activated microglia and astrocytes produce and release neuroexcitatory substances, including reactive oxygen species, nitric oxide, and inflammatory cytokines. ${ }^{9}$ Communication between neurons and glial cells increases the expression of pro-inflammatory and nociceptive signaling molecules. ${ }^{9}$ Calcitonin gene-related peptide (CGRP) released from neuronal cell bodies can stimulate glial cells to release inflammatory cytokines ${ }^{10}$ contributing to trigeminal sensitization. Inhibition of trigeminal neuronal-satellite glial cell signaling has been postulated as a target mechanism for anti-migraine therapy. ${ }^{11}$

\section{Adenosine 5' -triphosphate-mediated trigeminal sensitization}

Cortical spreading depression increases adenosine $5^{\prime}$ triphosphate (ATP) ${ }^{12}$ and other pro-inflammatory molecules in the cerebrospinal fluid. ${ }^{13}$ These may be involved in sensitization and activation of primary afferent sensory fibers in the trigeminal ganglion. ${ }^{13,14}$ Inflammatory and nociceptive effects of ATP are mediated by activation of P2X receptors, a member of a family of ligandgated ion channels expressed in peripheral sensory afferents and preferentially on nociceptive C-fibers. ${ }^{15,16}$ The $\mathrm{P} 2 \mathrm{X} 3$ receptors are located in brain areas that mediate nociception (e.g., the trigeminal nucleus and thalamus). ${ }^{16}$ $\mathrm{P} 2 \mathrm{X} 3$ receptors are the only ligand-gated channels ex- pressed exclusively by trigeminal neurons, making them a promising target for acute migraine treatment. ${ }^{16}$

ATP lowers the activation threshold of trigeminal neurons to $\mathrm{KCl}$ via $\mathrm{P} 2 \mathrm{X} 3$ receptors. ${ }^{17}$ Dihydroergotamine (DHE) downregulates P2X3-receptor expression and represses ATP-mediated trigeminal sensitization via activation of $\alpha 2$-adrenoreceptors. Primary cultures of trigeminal ganglion neurons were used to investigate the mechanism by which ATP causes sensitization of trigeminal neurons, and DHE represses this effect. CGRP release from trigeminal neurons did not significantly change from control levels when cultures were exposed to either $100 \mu \mathrm{M}$ ATP or $30 \mathrm{mM} \mathrm{KCl}$ alone. ATP exposure prior to $\mathrm{KCl}$ resulted in an approximately 4-fold increase in CGRP levels. The P2X3 purinergic receptor antagonist A-317491 significantly reduced CGRP release in response to ATP and $\mathrm{KCl}$. Therefore, the ATP-mediated threshold reduction for activation of trigeminal neurons by $\mathrm{KCl}$ is controlled, at least in part, by the $\mathrm{P} 2 \mathrm{X} 3$ receptor. Intracellular calcium was also significantly increased in response to the combination of ATP plus $\mathrm{KCl}$. This was also significantly suppressed by the $\mathrm{P} 2 \mathrm{X} 3$ receptor antagonist. Treatment with $10 \mu \mathrm{M}$ DHE alone did not repress the basal level of CGRP secretion, whereas preincubation with $10 \mu \mathrm{M}$ DHE for 30 minutes suppressed the stimulatory effect of ATP/KCl treatment on CGRP secretion. The suppressive effect of DHE was blocked by pretreatment with $3 \mathrm{nM} \alpha 2$-adrenoceptor antagonist yohimbine. DHE also significantly repressed the ATP plus $\mathrm{KCl}$-induced increase in intracellular calcium. This effect was also blocked by pretreatment with yohimbine.

Trigeminal cultures were stained using P2X3 antibodies. ${ }^{17} \mathrm{P} 2 \mathrm{X} 3$ staining was detected primarily in the cell membrane of trigeminal neurons. Treatment with DHE caused a large decrease in P2X3 staining. Pretreatment with yohimbine blocked the inhibitory effect of DHE on P2X3 membrane expression. This suggests that DHE decreases $\mathrm{P} 2 \mathrm{X} 3$ receptor protein in the cell membrane of trigeminal ganglion neurons by activating $\alpha 2$-adrenergic receptors.

\section{CGRP and treatment nonresponders}

Sarchielli et al. ${ }^{18}$ evaluated 10 migraineurs who responded and 10 who did not respond to rizatriptan and correlated poor acute migraine response with less trigeminal activation. Baseline plasma CGRP was lower in triptan nonresponders who also had a smaller change in CGRP after rizatriptan administration compared with responders. Cady et al. ${ }^{19}$ evaluated 22 migraineurs treating moderately severe migraine with rizatriptan and measured their salivary CGRP levels throughout the attack. When normalized to each subject's own baseline, CGRP levels were significantly elevated during the premonitory phase, with mild and moderate pain. CGRP decreased to baseline levels during migraine resolution. Salivary 
CGRP levels during each migraine stage were correlated with response to rizatriptan administered to treat a moderate to severe migraine. Among the 14 rizatriptan responders, CGRP increased significantly from baseline during premonitory and pain phases, returning to near baseline during resolution. In the eight nonresponders, CGRP did not change from baseline during any migraine phase. This suggests that only attacks associated with elevated salivary CGRP levels respond to rizatriptan. Triptan nonresponders may need to be treated with alternative mechanistic classes of acute medications.

\section{NOVEL ACUTE MIGRAINE FORMULATIONS}

Novel drugs and devices have been developed for acute migraine treatment to improve efficacy and tolerability. Currently available novel formulations include needle-free injectable sumatriptan and effervescent diclofenac. Orally inhaled DHE is being developed and has been proven to be effective in phase 3 clinical trials.

\section{Needle-free injectable sumatriptan}

Sumatriptan can be administered subcutaneously using a needle-free, injectable formulation, called DosePro (GlaxoSmithKline, Research Triangle Park, NC). Using this device, a small amount of compressed nitrogen gas forces $6 \mathrm{mg}$ of aqueous sumatriptan into subcutaneous tissues. The major advantage of this technology is improved patient acceptance. Needle-free, injectable sumatriptan is approved for the treatment of acute migraine and cluster headache.

Pharmacokinetic parameters are generally similar whether sumatriptan is administered using the needle-free Sumavel DosePro or the traditional STATdose (GlaxoSmithKline) injection, with a slightly earlier time to maximum concentration (Tmax) with the DosePro. ${ }^{20}$ Bioequivalence depends on injection site. A recent pharmacokinetics study showed bioequivalence using the standard needle and the needle-free DosePro when sumatriptan was administered in the thigh or abdomen (Table 1). ${ }^{21}$ Bioequivalence was not present when injecting into the arm with the needle-free system because of incomplete delivery. Due to similarities in bioequivalence between formulations for most injection sites, mi- graine relief was assumed to be equivalent between the two injectable formulations and efficacy trials were not required for approval.

Safety and tolerability have been compared between injectable formulations, with more local site reactions (immediate and nonpersistent bleeding, transient swelling, and transient erythema) using the needle-free system. ${ }^{20}$ A feasibility study showed that $98 \%$ of patients naïve to needle-free injection technology successfully injected sumatriptan in the thigh or abdomen on their first attempts. ${ }^{21}$ Postadministration bleeding, swelling, and erythema were more common with the needle-free system. One hour after dosing, injection-related side effects with the needle-free versus needle injection, respectively, included swelling in $61 \%$ versus $6 \%$ and erythema in $27 \%$ versus $7 \%$.

\section{Effervescent diclofenac}

Nonsteroidal anti-inflammatory drugs effectively treat migraine symptoms. In a sample of 24 healthy male volunteers, effervescent diclofenac demonstrated rapid absorption without lag time, with a 30-minute Tmax. ${ }^{22}$ Although pharmacokinetics will likely be delayed in migraineurs, a more rapidly absorbed oral formulation may provide better acute pain relief than traditional tablets.

Diclofenac potassium powder (approved by the Food and Drug Administration and marketed under the tradename Cambia), dissolved in water and taken orally, has a more rapid absorption than diclofenac tablets. ${ }^{23}$ In a randomized, controlled trial with 317 migraine patients, onset of analgesia (pain reduction significantly better than with placebo) occurred at 15 minutes with diclofenac potassium powder versus 60 minutes with diclofenac tablets. ${ }^{23}$ A 2-h pain-free response was achieved by $25 \%$ of patients using diclofenac potassium powder, $19 \%$ using diclofenac tablets, and $12 \%$ using placebo (FIG. 1). ${ }^{23}$ Both diclofenac formulations were significantly better than the placebo, with the powder also superior to diclofenac tablets $(p<0.01)$. A 24 -h sustained pain-free response occurred for $22 \%$ of patients using diclofenac powder versus $15 \%$ using the tablet and $9 \%$ using placebo. These differences were also statistically significant $(p<0.05)$.

Table 1. Pharmacokinetics of $6 \mathrm{mg}$ Sumatriptan Using Needle or Needle-Free Subcutaneous Injections ${ }^{21}$

\begin{tabular}{|c|c|c|c|c|c|c|}
\hline & \multicolumn{6}{|c|}{ Injection site } \\
\hline & \multicolumn{2}{|c|}{ Abdomen } & \multicolumn{2}{|c|}{ Thigh } & \multicolumn{2}{|c|}{ Arm } \\
\hline & Needle & Needle-free & Needle & Needle-free & Needle & Needle-free \\
\hline Mean Cmax (ng/mL) & 87.3 & 78.6 & 68.3 & 71.9 & 83.4 & 76.6 \\
\hline Median Tmax (h) & 0.20 & 0.20 & 0.23 & 0.20 & 0.18 & 0.15 \\
\hline AUC (ng h/mL) & 104.0 & 95.3 & 101.0 & 92.9 & 99.3 & 82.5 \\
\hline
\end{tabular}

$\mathrm{AUC}=$ area under the curve; $\mathrm{Cmax}=$ maximum plasma concentration; Tmax $=$ time to maximum concentration. 


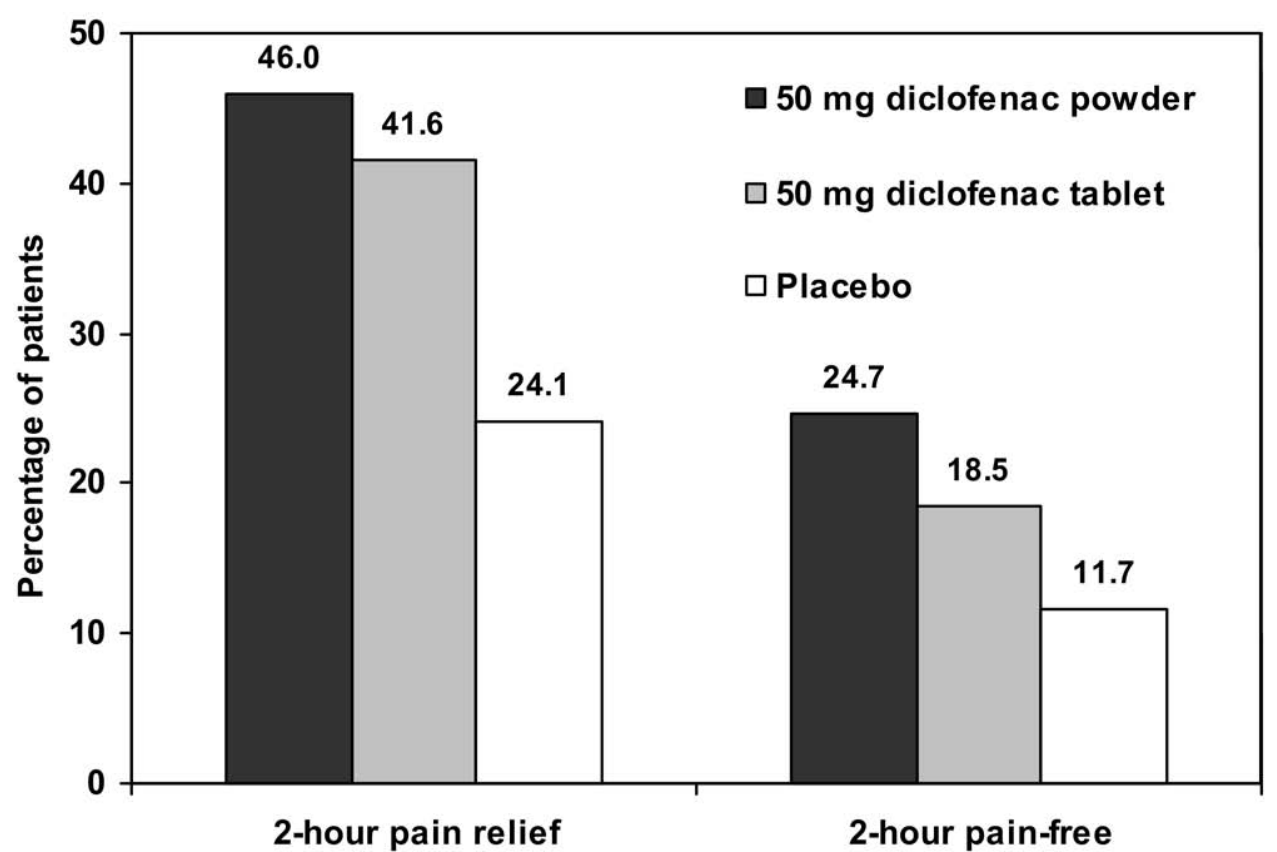

FIG. 1. Diclofenac potassium powder efficacy in migraineurs. ${ }^{23}$

Orally inhaled DHE

Lung alveoli provide a large, absorptive potential surface for drug absorption, offering ready access to the systemic circulation. ${ }^{24,25}$ Inhalation administration can rapidly and predictably achieve effective drug plasma concentrations through alveolar delivery. ${ }^{26}$ The best absorption occurs when drugs reach the deep alveoli, with lung deposition dependent on qualities of both the drug and the lung. ${ }^{24}$ Major drug determinants of absorption include small molecular size and lipophilicity. ${ }^{24}$ Respi-

\section{$\square$ Orally inhaled DHE (N=395) $\square$ Placebo $(\mathrm{N}=397)$}

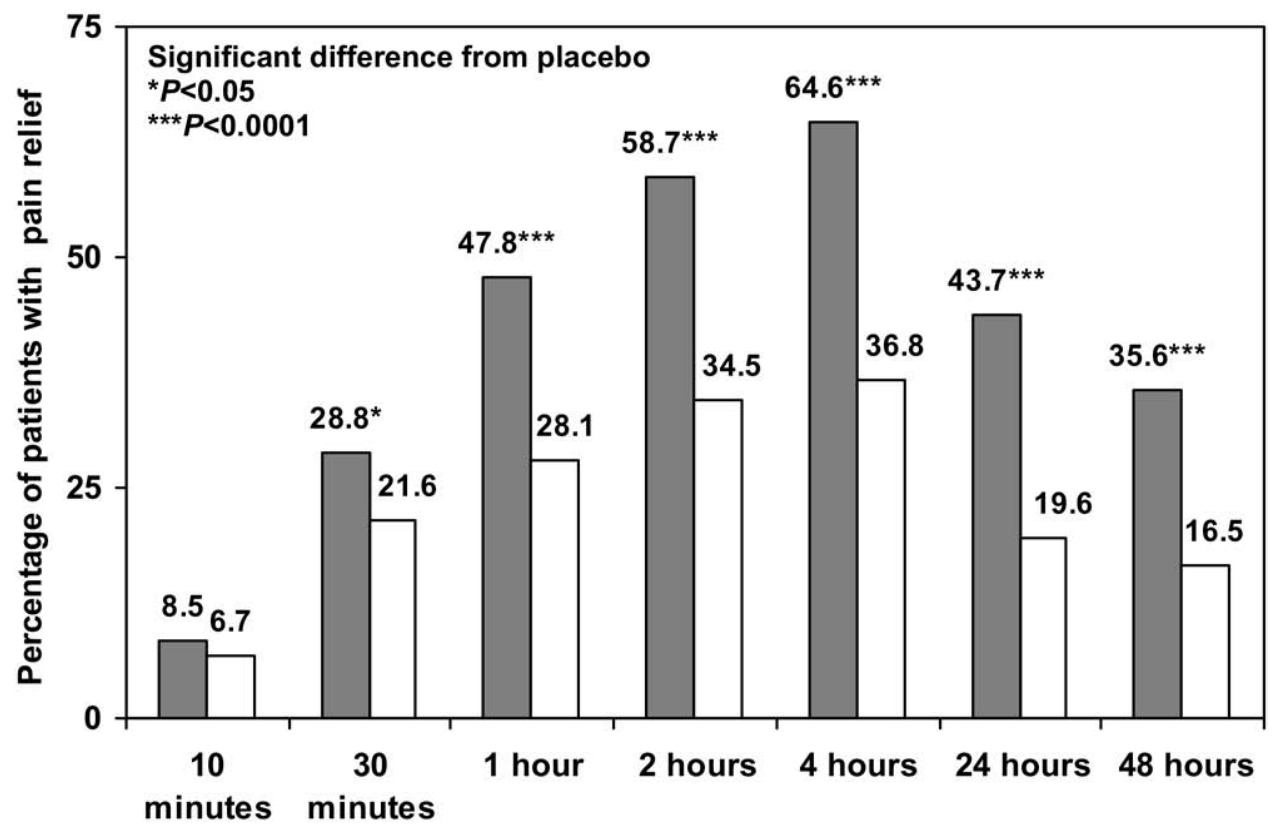

Time post-treatment

FIG. 2. Migraine pain relief with orally inhaled dihydroergotamine DHE. ${ }^{30}$ Pain relief data were obtained for the first 4 hours, with 24 -hour and 48-hour relief describing sustained relief from 2 to 24 and 2 to 48 hours, respectively. 
ratory status (including anatomy, age, gender, lung volume, and lung health) also affects drug absorption. ${ }^{24}$ Inhalation administration allows rapid systemic drug delivery, similar to that typically achieved through intravenous (IV) therapy.

A newly developed, orally inhaled formulation of DHE (MAP0004) delivers the drug using a pressurized, metered dose inhaler. Orally inhaled DHE has a Tmax of approximately 10 minutes, similar to IV DHE. ${ }^{27}$ Mean peak plasma DHE concentrations are much lower with orally inhaled DHE than with IV DHE, suggesting a lower potential for adverse events. ${ }^{27}$ A study comparing receptor binding with orally inhaled DHE and IV DHE showed similar binding with both formulations to receptors important for anti-migraine effect (e.g., 5- $\mathrm{HT}_{1 \mathrm{~A}}$, $5-\mathrm{HT}_{1 \mathrm{~B}}$, and $5-\mathrm{HT}_{1 \mathrm{D}}$ receptors). ${ }^{28}$ Lower binding of the inhaled formula to catecholamine receptors were postulated to contribute to its better tolerability.

Nonsmoking adult migraineurs $(\mathrm{n}=86)$ were randomized to acute migraine treatment with orally inhaled DHE ( 0.5 or $1.0 \mathrm{mg}$ systemic equivalent dose) or matching placebo. ${ }^{29}$ Reduction in pain from moderate or severe to mild or no pain occurred in 10 minutes after treatment for one in three patients treated with $0.5 \mathrm{mg}$ orally inhaled DHE, one in five treated with $1 \mathrm{mg}$, and none treated with placebo. This difference was statistically significant for the $0.5 \mathrm{mg}$ dose $(p=0.02)$, with a trend for the $1 \mathrm{mg}$ dose $(p=0.06)$. Two-hour pain relief occurred for $72 \%$ treated with $0.5 \mathrm{mg}$ orally inhaled DHE, $65 \%$ with $1 \mathrm{mg}$, and $33 \%$ with placebo. Pain freedom was first seen after 10 minutes with $1 \mathrm{mg}$ orally inhaled DHE, 30 minutes after $0.5 \mathrm{mg}$ orally inhaled DHE, and 60 minutes after placebo. After $2 \mathrm{~h}, 35$ to $44 \%$ of patients treated with orally inhaled DHE were painfree compared with $7 \%$ treated with placebo. A pain-free response was sustained at 24 and $48 \mathrm{~h}$, respectively, in $38 \%$ and $37 \%$ of patients treated with $0.5 \mathrm{mg}$ orally inhaled DHE, $22 \%$ and $13 \%$ with $1 \mathrm{mg}$, and $7 \%$ with placebo at both time points.

New phase 3 trial data showed that early pain relief is significantly better with orally inhaled DHE than placebo, with a significant difference realized 30 minutes after dosing (FIG. 2). ${ }^{30}$ Nearly half of the treated patients achieved pain relief within 1 hour of treatment, with sustained pain relief for at least 24 hours achieved by more than $40 \%$. Adverse events were infrequent with orally inhaled DHE versus placebo: nausea (4.5\% vs. $2.0 \%$ ), cough $2.5 \%$ versus $1.2 \%$, bad taste (6.4\% vs. $1.7 \%$ ).

\section{CONCLUSIONS}

Despite the development and availability of migrainespecific medications, only $19 \%$ of migraineurs use triptans for acute therapy, ${ }^{1}$ and triptan satisfaction is modest at best. ${ }^{2}$ Although patients prefer oral adminis- tration, gastric stasis limits the usefulness of this route of administration.

New data on migraine pathogenesis focus on the role of trigeminal activation and CGRP release. More robust changes in CGRP seem to predict triptan response. ${ }^{18,19}$ Small studies have shown that a substantial minority of migraineurs lack an adequate CGRP response to achieve triptan response. ${ }^{19}$ This may at least partially explain why one in three migraineurs may not be a triptan responder. ${ }^{5}$ DHE blocks trigeminal sensitization via a different mechanism. Patients who fail to achieve success with currently available triptans may need to consider alternative formulations and nontriptan therapies.

Novel acute migraine formulations may improve clinical outcomes. Currently available novel agents include needle-free injectable sumatriptan and effervescent diclofenac. Orally inhaled DHE has shown promise in phase 3 trials and will likely be available soon for migraine patients. New acute migraine formulations have been shown to achieve more desirable treatment targets of quicker and more complete relief ${ }^{3}$ by providing more rapid onset of action, sustained anti-migraine effect, and improved consistency of response.

Acknowledgments: Technical support was provided by Shashidhar H. Kori, Ph.D., MAP Pharmaceuticals, and Dawn A. Marcus, M.D., medical writer. Funding was provided by MAP Pharmaceuticals.

\section{REFERENCES}

1. Bigal ME, Borucho S, Serrano D, Lipton RB. The acute treatment of episodic and chronic migraine in the USA. Cephalalgia 2009; 29:891-897.

2. Revicki DA, Kimel M, Beusterien K, et al. Validation of the Revised Patient Perception of Migraine Questionnaire: measuring satisfaction with acute migraine treatment. Headache 2006;46: 240-252.

3. Davies GM, Santanello N, Lipton R. Determinants of patient satisfaction with migraine therapy. Cephalalgia 2000;20:554-560.

4. Diener HC, Limmroth V. Advances in pharmacological treatment of migraine. Expert Opin Investig Drugs 2001;10:1831-1845.

5. Velati D, Viana M, Cresta S, et al. 5-hydroxytryptamine1B receptor and triptan response in migraine, lack of association with common polymorphisms. Eur J Pharmacol 2008;580:43-47.

6. Lipton RB, Hamelsky BA, Dayno JM. What do patients with migraine want from acute migraine treatment? Headache 2002; 42(suppl 1):S3-S9.

7. Aurora S, Kori S, Barrodale P, Nelsen A, McDonald S. Gastric stasis occurs in spontaneous, visually induced, and interictal migraine. Headache 2007;47:1443-1446.

8. Dodick D, Silberstein S. Central sensitization theory of migraine: clinical implications. Headache 2006;46(suppl 4):S182-S191.

9. Bartley J. Could glial activation be a factor in migraine? Med Hypotheses 2009;72:255-257.

10. Thalakoti S, Patil VV, Damodaram S, et al. Neuron-glia signaling in trigeminal ganglion: implications for migraine pathology. Headache 2007;47:1008-1025.

11. Damodaram S, Thalakoti S, Freeman SE, Garrett FG, Durham PL. Tonabersat inhibits trigeminal ganglion neuronal-satellite glial cell signaling. Headache 2009;49:5-20.

12. Schock SC, Munyao N, Yakubchyk Y, et al. Cortical spreading depression releases ATP into the extracellular space and purinergic receptor activation contributes to the induction of ischemic tolerance. Brain Res 2007;1168:129-138. 
13. Bolay H, Reuter U, Dunn AK, Huang Z, Boas DA, Moskowitz MA. Intrinsic brain activity triggers trigeminal meningeal afferents in a migraine model. Nat Med 2002;8:136-142.

14. Moskowitz MA. Defining a pathway to discovery from bench to bedside: the trigeminovascular system and sensitization. Headache 2008;48:688-690.

15. Bie BH, Zhang YH, Zhao ZQ. Inhibition of $\mathrm{P} 2 \mathrm{X}$ receptor-mediated inward current by protein kinase $\mathrm{C}$ in small-diameter dorsal root ganglion neurons of adult rats. Neurosci Bull 2009;25:179-186.

16. Burnstock G. Purinergic receptors and pain. Curr Pharm Des 2009; 15:1717-1735.

17. Masterson C, Durham P. DHE repression of ATP-mediated sensitization of trigeminal ganglion neurons involves activation of $\alpha 2$ adrenergic receptors. Cephalalgia 2010 (in press).

18. Sarchielli P, Pini LA, Zanchin G, et al. Clinical-biochemical correlates of migraine attacks in rizatriptan responders and nonresponders. Cephalalgia 2006;26:257-265.

19. Cady RK, Vause CV, Ho TW, Bigal ME, Durham PL. Elevated saliva calcitonin gene-related peptide levels during acute migraine predict therapeutic response to rizatriptan. Headache 2009;49: $1258-1266$

20. Farr SJ, Linn LS, Shabe P, Newman E, Chandler P. Pharmacokinetics, bioequivalence, and safety of the sumatriptan DosePro needle-free system vs. IMITREX STATdose in healthy adults. Presented at the $50^{\text {th }}$ Annual Scientific Meeting of the American Headache Society; June 28, 2008; Boston.

21. Brandes JL, Cady RK, Freitag FG, et al. Needle-free subcutaneous sumatriptan (Sumavel DosePro): bioequivalence and ease of use. Headache 2009;49:1435-1444.
22. Terhaag B, Hoffmann A, Barkworth M, Vens-Cappell B. Bioavailability of a new effervescent tablet of diclofenac. Int J Clin Pharmacol Ther 2000;38:546-551.

23. Diener HC, Montagna P, Gács G, et al. Efficacy and tolerability of diclofenac potassium sachets in migraine: a randomized, doubleblind, cross-over study in comparison with diclofenac potassium tablets and placebo. Cephalalgia 2005;26:537-547.

24. Gonda I. Systemic delivery of drugs to humans via inhalation. J Aerosol Med 2006;19:47-53.

25. Siekmeier R, Scheuch G. Systemic treatment by inhalation of macromolecules - principles, problems, and examples. J Physiol Pharmacol 2008;59(suppl 6):53-79.

26. Patton JS, Byron PR. Inhaling medicines: delivering drugs to the body through the lungs. Nat Rev Drug Discov 2007;6:67-74.

27. Shrewsbury SB, Cook RO, Taylor G, Edwards C, Ramadan NM. Safety and pharmacokinetics of dihydroergotamine mesylate administered via a novel $\left(\mathrm{Tempo}^{\mathrm{TM}}\right)$ inhaler. Headache 2008;48: 355-367.

28. Cook RO, Shrewsbury SB, Ramadan NM. Reduced adverse event profile of orally inhaled DHE (MAP00004) vs. IV DHE: potential mechanism. Headache 2009;49:1423-1434.

29. Aurora SK, Rozen TD, Kori SH, Shrewsbury SB. A randomized, double blind, placebo-controlled study of MAP0004 in adult patients with migraine. Headache 2009;49:826-837.

30. Silberstein S, Kori SH, Aurora S, et al. LEVADEX, a novel orally inhaled treatment for acute migraine: efficacy and tolerability results of a Phase 3 study. Presented at the 14th International Headache Congress; September 12, 2009; Philadelphia. 